\title{
Avaliação clínica e objetiva da deglutição em crianças com paralisia cerebral
}

\author{
Clinical and instrumental swallowing evaluation in children \\ with cerebral palsy
}

\author{
Moisés Andrade dos Santos de Queiroz ${ }^{1}$, Izabella Santos Nogueira de Andrade², \\ Renata Cavalcante Barbosa Haguette ${ }^{3}$, Erik Frota Haguette ${ }^{2}$
}

\begin{abstract}
RESUMO
Objetivo: Investigar as principais manifestações disfágicas na avaliação clínica e objetiva da deglutição em crianças com paralisia cerebral. Métodos: Estudo transversal com amostra de 50 crianças com paralisia cerebral. A coleta de dados consistiu na realização das avaliações clínica fonoaudiológica e videoendoscópica da deglutição para a deteç̧ão das principais manifestações disfágicas presentes nas fases preparatória oral, oral e faríngea da deglutição, em diferentes consistências alimentares e tipos de paralisia cerebral. Resultados: Não houve influência significativa da idade e do tipo de paralisia cerebral sobre a maior presença de manifestações disfágicas. As principais manifestações disfágicas na avaliação clínica foram: escape anterior de líquidos (70\%); tosse na deglutição de líquidos (60\%); diminuição da formação do bolo alimentar sólido (57,9\%); presença de resíduos alimentares na cavidade oral após a deglutição de sólidos $(47,4 \%)$; aumento do trânsito oral de pastosos grossos $(47,1 \%)$ e presença da voz molhada após a deglutição de líquidos (30\%). As principais manifestações disfágicas na avaliação objetiva foram: aumento do trânsito faríngeo de pastosos grossos (79\%); escape posterior de líquidos (77,5\%); presença de resíduos alimentares nas estruturas faríngeas após a deglutição de pastosos grossos (58,8\%); penetração laríngea de líquidos (37,5\%) e aspiração traqueal de líquidos (20\%). A sensibilidade laríngea esteve alterada em apenas $16 \%$ da amostra. Conclusão: As avaliações clínica e objetiva da deglutição são complementares e essenciais no diagnóstico das disfagias orofaríngeas em crianças com paralisia cerebral.
\end{abstract}

Descritores: Deglutição; Paralisia cerebral/complicações; Transtornos da deglutição/diagnóstico; Faringe/fisiopatologia; Criança

\section{INTRODUÇÃO}

A dinâmica da deglutição é composta por fases (preparatória oral, oral, faríngea e esofágica) intrinsecamente relacionadas que têm como objetivo transportar, de maneira eficaz, os alimentos da cavidade oral ao estômago ${ }^{(1)}$.

Comprometimentos em qualquer fase e/ou entre as fases da deglutição caracterizam as disfagias ${ }^{(2)}$, que, na infância, são frequentes nos casos de paralisia cerebral (PC), acarretando riscos de desnutrição, desidratação e infecções pulmonares ${ }^{(3)}$.

Trabalho realizado no Setor de Terapia Ocupacional e Ambulatório de Disfagia do Núcleo de Atenção Médica Integrada (NAMI), Universidade de Fortaleza - UNIFOR - Fortaleza (CE), Brasil.

(1) Programa de Pós-graduação (Mestrado) em Ciência Médicas da Faculdade de Medicina, Universidade Federal do Ceará - UFC - Fortaleza (CE), Brasil.

(2) Curso de Fonoaudiologia, Universidade de Fortaleza - UNIFOR - Fortaleza (CE), Brasil.

(3) Programa de Pós-graduação (Doutorado) em Saúde Coletiva da Faculdade de Medicina, Universidade Federal do Ceará - UFC - Fortaleza (CE), Brasil. Endereço para correspondência: Moisés Andrade dos Santos de Queiroz. R. 1044, 16, Conjunto Ceará, Fortaleza (CE), Brasil, CEP: 60532-820. E-mail: moisesandrade@live.com

Recebido em: 27/6/2010; Aceito em: 16/9/2010
Os métodos mais utilizados para a investigação das disfagias são a avaliação clínica fonoaudiológica (ACF) e os exames objetivos, como a avaliação videoendoscópica da deglutição (VED) ${ }^{(4-6)}$.

$\mathrm{Na} A C F$, realiza-se criteriosa história clínica com avaliação morfofuncional da deglutição, especialmente das fases preparatória oral e oral, visando prevenir e/ou reduzir os riscos à saúde ${ }^{(4)}$.

A VED consiste em uma nasofibrolaringoscopia tradicional, com o oferecimento de alimentos tingidos com corantes, possibilitando a investigação da fase faríngea da deglutição, a qual apresenta o caminho concomitante entre vias respiratória e digestiva superior ${ }^{(5,6)}$.

Com apoio nos achados da ACF e VED, realizam-se adequações na dieta alimentar da criança com PC, como a escolha de volumes, consistências e manobras compensatórias apropriadas para a alimentação, no intuito de prevenir a ocorrência de manifestações disfágicas ${ }^{(6,7)}$.

Ante o exposto, este estudo tem como objetivo investigar as principais manifestações disfágicas na avaliação clínica e objetiva da deglutição em crianças com PC. 


\section{MÉTODOS}

Anteriormente à execução deste estudo, o projeto foi avaliado e aprovado pelo Comitê de Ética em Pesquisa da Universidade de Fortaleza (UNIFOR), sob o número 105/09. Os responsáveis pelas crianças participantes do estudo assinaram Termo de Consentimento Livre e Esclarecido, conforme prescrito na Resolução n ${ }^{\circ}$ 196/96, do Conselho Nacional de Saúde (CNS).

Realizou-se um estudo quantitativo, transversal e observacional, em crianças diagnosticadas com PC, no Setor de Terapia Ocupacional e Ambulatório de Disfagia do Núcleo de Atenção Médica Integrada (NAMI), no período de fevereiro a outubro do ano de 2009.

Foram incluídas as crianças com diagnóstico de PC, independentemente dos fatores pré, peri e pós-natais e do tipo de PC. Foram excluídas do estudo as crianças que apresentaram anomalias estruturais de orofaringolaringe e/ou impossibilidade de alimentar-se por via oral.

A amostra foi composta por 50 crianças com PC, com média de idade de 1 ano e 6 meses, variando entre 1 e 5 anos de idade cronológica, sendo 27 do gênero masculino e 23 do gênero feminino. Destas, 36\% (18/50) foram classificadas como atetóides e $64 \%$ (32/50) como espásticas.

Os instrumentos empregados na coleta de dados consistiram dos protocolos de ACF e VED, utilizados no Setor de Terapia Ocupacional e Ambulatório de Disfagia do NAMI.

Inicialmente as crianças foram submetidas à $\mathrm{ACF}$, composta por história clínica infantil, incluindo informações sobre intercorrências, tratamentos, condições alimentares e respiratórias. Esta avaliação envolveu, ainda, a análise das estruturas orofaciais, como lábios, bochechas, língua, palato, mandíbula e oclusão, bem como a postura, o tônus, a mobilidade e a sensibilidade.

A observação da alimentação ocorreu com o oferecimento de $40 \mathrm{ml}$ de líquido (água), $40 \mathrm{ml}$ de pastoso fino (iogurte Nestlé ${ }^{\circledast}$ ), $40 \mathrm{ml}$ de pastoso grosso (iogurte Nestlé ${ }^{\circledast}$ acrescido de duas colheres de $10 \mathrm{ml}$ de espessante alimentar instantâneo Thick \& Easy ${ }^{\circledR}$ ) e sólido (dois biscoitos Maizena ${ }^{\circledR}$ ). Os alimentos foram oferecidos pelo responsável em ordem crescente de dificuldade, com posicionamento e utensílios habituais de alimentação. Não foram ofertadas as consistências em que as crianças não eram habituadas a se alimentar ou que apresentavam aspiração laríngea previamente diagnosticada.

Após a ACF, realizou-se a VED, a qual foi interpretada pelo otorrinolaringologista e pela fonoaudióloga do Ambulatório de Disfagia. Os exames foram realizados utilizando-se o nasofibroscópio Olympus ${ }^{\circledR} \mathrm{P} 4$, acoplado à câmera de vídeo Wolf, ao DVD player e monitor colorido Samsung®, para a gravação dos exames em mídias de DVD.

As crianças permaneceram em posição similar à ACF. O nasofibroscópio foi introduzido na cavidade nasal, sem a utilização de anestésicos ou vasoconstritores tópicos. O aparelho foi avançado em direção à rinofaringe, onde se avaliou a morfofuncionalidade do véu palatino durante a fonação e/ou deglutição de saliva. $\mathrm{O}$ aparelho seguiu em direção à orofaringe para a observação da base da língua, faringe, verificando a presença de estase salivar em valéculas e/ou recessos piriformes e laringe, observando a mobilidade das pregas vocais.

Após a avaliação estrutural, iniciou-se a avaliação funcional da deglutição, com o oferecimento dos alimentos consumidos na ACF, porém tingidos com corante inorgânico azul, para facilitar a visualização do alimento durante o exame.

Observou-se o controle do alimento na boca, avaliando a capacidade de controle do alimento sob comando. O trânsito orofaríngeo foi avaliado de acordo com as variáveis: início da deglutição faríngea, clearance faríngeo e proteção das vias aéreas inferiores.

A sensibilidade laríngea foi testada por meio do toque com a extremidade distal do aparelho nas pregas vocais, aritenóides e/ou bandas ventriculares, permitindo a observação da ocorrência de adução glótica e tosse reflexa.

Foram consideradas as variáveis para a ACF e VED a seguir descritas:

- Manifestações disfágicas na ACF:

- escape anterior - definido como a ocorrência de escorrimento do alimento pelos lábios, após sua captação deste; - aumento do trânsito oral - definido quando o tempo entre a formação completa do bolo alimentar e o início da elevação do complexo hiolaríngeo ultrapassa quatro segundos;

- diminuição da formação do bolo alimentar - definida na presença de bolo alimentar não coeso na cavidade oral;

- estase alimentar oral - definida como o acúmulo de alimento em vestíbulo anterior, lateral, assoalho da cavidade oral e/ou superfície da língua após a deglutição;

- tosse - definida como a presença de tosse reflexa durante ou após a deglutição; e

- voz molhada - definida como a ocorrência de som borbulhante durante a fonação pós deglutição.

- Manifestações disfágicas na VED:

- escape posterior - definido como a ocorrência de escorrimento do alimento da cavidade oral para a hipofaringe antes do mecanismo de ejeção da língua;

- aumento do trânsito faríngeo - definido quando o tempo entre o início das fases faríngea e esofágica da deglutição ultrapassa dois segundos;

- estase alimentar faríngea - definida como o acúmulo de alimento em valéculas e/ou recessos piriformes após três deglutições;

- penetração laríngea - definida como a ocorrência de entrada de alimento na laringe até o nível das pregas vocais, caracterizada em antes, durante ou após o início da fase faríngea;

- aspiração traqueal - definida como a ocorrência de entrada de alimento na laringe abaixo do nível das pregas vocais, caracterizada em antes, durante ou após o início da fase faríngea; e

- alteração na sensibilidade laríngea - definida como a ausência de adução glótica e tosse reflexa ao toque com a extremidade distal do aparelho nas pregas vocais, aritenóides e/ou bandas ventriculares, permitindo a observação da ocorrência de adução glótica e tosse reflexa.

Após a análise dessas variáveis, observaram-se as principais manifestações disfágicas em cada avaliação, nas diferentes consistências alimentares, idades e tipos de PC.

Para a análise estatística entre as variáveis de cada grupo, 
optou-se por empregar os testes ANOVA e Igualdade de Duas Proporções, realizados mediante o software SPSS v16, considerando o nível de significância $\mathrm{p} \leq 0,05$.

\section{RESULTADOS}

Todas as crianças com PC (100\% - 50/50) apresentaram alguma manifestação disfágica durante a ACF e VED, sendo considerado um dado significativo ( $\mathrm{p} \leq 0,001)$.

Utilizou-se o ANOVA na comparação entre as idades, em meses, e a presença de manifestações disfágicas durante a ACF e VED. Constatou-se que não houve influência significativa da idade influenciando na presença da disfagia orofaríngea em crianças com PC.

No que concerne à função de deglutição, não se observou diferença significativa entre o tipo de PC e a presença de manifestações disfágicas nas avaliações.

Durante a ACF e VED, 80\% (40/50) das crianças com PC se alimentaram com líquido, $52 \%(26 / 50)$ com pastoso fino, $34 \%$ (17/50) com pastoso grosso e 38\% (19/50) com sólidos. Ressalta-se que não foram administradas as consistências que a criança não consome habitualmente.

As principais manifestações disfágicas na $\mathrm{ACF}$, em ordem decrescente de ocorrência, foram: escape anterior de líquidos; tosse antes, durante ou após a deglutição de líquidos; diminuição da formação do bolo alimentar sólido; presença de resíduos alimentares na cavidade oral após a deglutição de sólidos; aumento do trânsito oral de pastosos grossos e presença da voz molhada após a deglutição de líquidos (Tabela 1).
Quanto às principais manifestações disfágicas na VED, em ordem decrescente de ocorrência, foram encontradas: aumento do trânsito faríngeo de pastosos grossos; escape posterior de líquidos; presença de resíduos alimentares nas estruturas faríngeas após a deglutição de pastosos grossos; penetração laríngea de líquidos e aspiração traqueal de líquidos antes, durante ou após a deglutição (Tabela 2).

A alteração na sensibilidade laríngea foi observada em apenas $16 \%(8 / 50)$ das crianças com PC.

\section{DISCUSSÃO}

A PC pode ser definida como uma encefalopatia crônica não progressiva, porém mutável, que acarreta desordem do tônus postural e movimento decorrente de um comprometimento do cérebro imaturo. Pode ser classificada, de acordo com o local da lesão e alteração de tônus muscular, em espástica, atetóide, atáxica ou mista ${ }^{(8)}$.

Independentemente de sua classificação clínica, o desenvolvimento neuropsicomotor da criança com PC acompanha alterações motoras globais e orais que manifestam transtornos da deglutição, os quais são agravados na presença de retardo mental $^{(9-11)}$.

Tais alterações justificam a semelhança encontrada nas manifestações disfágicas nos dois grupos de PC, já que uma deglutição eficiente é dependente dos sistemas motores global e oral ${ }^{(12,13)}$. Outro aspecto que influenciou na não-significância das diferenças entre o tipo de PC e o maior comprometimento da deglutição foi o tamanho da amostra; porém, nos valores brutos, verificou-se que as crianças com PC atetóide apresen-

Tabela 1. Manifestações disfágicas na avaliação clínica fonoaudiológica em crianças com paralisia cerebral

\begin{tabular}{|c|c|c|c|c|c|c|c|c|}
\hline \multirow{2}{*}{ Manifestação disfágica } & \multicolumn{2}{|c|}{ Líquida $(\mathrm{n}=40)$} & \multicolumn{2}{|c|}{ Pastosa fina $(n=26)$} & \multicolumn{2}{|c|}{ Pastosa grossa $(n=17)$} & \multicolumn{2}{|c|}{ Sólida $(n=19)$} \\
\hline & n (\%) & Valor de $p$ & n (\%) & Valor de $p$ & $\mathrm{n}(\%)$ & Valor de $p$ & $\mathrm{n}(\%)$ & Valor de $p$ \\
\hline Escape anterior & $28(70)$ & $<0,001^{\star}$ & $18(69,2)$ & $0,006^{*}$ & $10(58,8)$ & 0,303 & $13(68,4)$ & $0,023^{*}$ \\
\hline Aumento do trânsito oral & $12(30)$ & $<0,001^{*}$ & $11(42,3)$ & 0,267 & $8(47,1)$ & 0,732 & $6(31,6)$ & $0,023^{*}$ \\
\hline $\begin{array}{l}\text { Diminuição da formação do bolo } \\
\text { alimentar }\end{array}$ & $12(30)$ & $<0,001^{\star}$ & $11(42,3)$ & 0,267 & $9(52,9)$ & 0,732 & $11(57,9)$ & 0,330 \\
\hline Resíduo alimentar & $4(10)$ & $<0,001^{*}$ & $5(19,2)$ & $<0,001^{*}$ & $7(41,2)$ & 0,303 & $9(47,4)$ & 0,746 \\
\hline Tosse & $24(60)$ & 0,074 & $10(38,5)$ & 0,096 & $6(35,3)$ & 0,086 & $6(31,6)$ & $0,023^{*}$ \\
\hline Voz molhada & $12(30)$ & $<0,001^{*}$ & $6(23,1)$ & $<0,001^{*}$ & $5(29,4)$ & $0,016^{*}$ & $5(26,3)$ & $0,004^{*}$ \\
\hline
\end{tabular}

* Valores significantes $(p \leq 0,05)$ - Teste Igualdade de Duas Proporções

Tabela 2. Manifestações disfágicas na avaliação videoendoscópica da deglutição em crianças com paralisia cerebral

\begin{tabular}{|c|c|c|c|c|c|c|c|c|}
\hline \multirow{2}{*}{ Manifestação disfágica } & \multicolumn{2}{|c|}{ Líquida $(n=40)$} & \multicolumn{2}{|c|}{ Pastosa fina $(n=26)$} & \multicolumn{2}{|c|}{ Pastosa grossa $(n=17)$} & \multicolumn{2}{|c|}{ Sólida $(n=19)$} \\
\hline & $\mathrm{n}(\%)$ & Valor de $p$ & $\mathrm{n}(\%)$ & Valor de $p$ & $\mathrm{n}(\%)$ & Valor de $p$ & $\mathrm{n}(\%)$ & Valor de $p$ \\
\hline Escape posterior & $31(77,5)$ & $<0,001^{*}$ & $18(69,2)$ & $0,006^{*}$ & $9(52,9)$ & 0,355 & $7(36,8)$ & $0,025^{*}$ \\
\hline Aumento do trânsito faríngeo & $11(27,5)$ & $<0,001^{*}$ & $13(50)$ & 0,279 & $13(76,5)$ & $0,009^{*}$ & $15(79)$ & $0,002^{*}$ \\
\hline $\begin{array}{l}\text { Resíduo alimentar nas estruturas } \\
\text { faríngeas }\end{array}$ & $6(15)$ & $<0,001^{*}$ & $6(23,1)$ & $<0,001^{*}$ & $10(58,8)$ & 0,790 & $11(57,9)$ & 0,330 \\
\hline Penetração laríngea & $15(37,5)$ & $<0,001^{*}$ & $6(23,1)$ & $<0,001^{*}$ & $2(11,7)$ & $<0,001^{*}$ & $1(5,2)$ & $<0,001^{*}$ \\
\hline Aspiração traqueal & $8(20)$ & $<0,001^{*}$ & $3(11,5)$ & $<0,001^{*}$ & $1(5,9)$ & $<0,001^{*}$ & $1(5,2)$ & $<0,001^{*}$ \\
\hline
\end{tabular}

* Valores significantes $(p \leq 0,05)$ - Teste Igualdade de Duas Proporções 
taram menor quantidade de manifestações disfágicas nas duas avaliações realizadas.

O escape anterior de líquido pode estar relacionado ao vedamento labial ineficiente e às alterações sensoriais na cavidade oral ${ }^{(14)}$. Destaca-se que estes achados influenciam no desempenho das estruturas orais responsáveis pela manipulação, organização e ejeção do bolo alimentar, ocasionando o escape posterior de líquidos, o que pode resultar na entrada do alimento na via aérea desprotegida ${ }^{(14,15)}$.

As alterações no tônus e na mobilidade de lábios, bochechas e língua contribuíram para o aumento do trânsito oral e para a diminuição da formação do bolo alimentar. Estudos ${ }^{(14-17)}$ relatam que o tempo do trânsito oral, que é regulado na área do córtex cerebral responsável pela movimentação voluntária, encontra-se aumentado em crianças com PC em razão das desordens motoras e cognitivas que estas apresentam.

As dificuldades na formação e ejeção do bolo alimentar para a faringe e a quebra da pressão intraoral favorecem a presença de resíduos alimentares na cavidade oral após a deglutição ${ }^{(18)}$. Somadas aos prejuízos da musculatura faríngea, essas alterações contribuem para o aumento do trânsito faríngeo e, consequentemente, para a presença de resíduos alimentares nas estruturas faríngeas de valéculas e recessos piriformes ${ }^{(18,19)}$.

Indivíduos com disfagia orofaríngea neurogênica, frequentemente apresentam lentidão e falta de controle das estruturas orais relacionadas às fases oral e preparatória da deglutição, fato que acarreta resíduos alimentares nas estruturas faríngeas, manifestações disfágicas associadas à penetração laríngea e aspiração traqueal após a deglutição ${ }^{(14-21)}$.

A sensibilidade laríngea esteve presente na maioria das crianças com PC, sendo considerado um achado positivo neste estudo, em razão da possibilidade da ativação do mecanismo de tosse reflexa, que favorece a limpeza da via aérea ${ }^{(22)}$.

Quanto às consistências alimentares, os resultados demonstraram que as alterações mais associadas à consistência líquida foram o escape anterior e posterior do alimento, além da entrada de substâncias na via aérea. A presença de resíduos alimentares em cavidade oral e estruturas faríngeas, assim como o aumento do trânsito oral e faríngeo, foram observados com maior frequência no aumento da consistência do bolo alimentar.

Geralmente, as consistências líquidas e sólidas são as de mais difícil controle pela criança com PC, sendo a pastosa considerada a mais indicada para o oferecimento à criança, em virtude das melhores informações sensoriais que esta oferece $^{(21,23,24)}$.

A consistência sólida foi a menos consumida pelas crianças deste estudo. Este fato deve ter ocorrido em razão da maior exigência na manipulação, organização e formação do bolo alimentar e ao receio dos responsáveis em introduzir alimentos de texturas diferentes ${ }^{(21,25)}$.

Esses dados demonstram a hierarquia em relação às manifestações disfágicas e às diferentes consistências alimentares, evidenciando a importância do estudo das disfagias mediante as avaliações clínica e objetiva da deglutição.

Destaca-se o fato de que houve correlação entre as avaliações clínica e objetiva da deglutição, contribuindo para os questionamentos referentes à presença de aspiração traqueal silenciosa em crianças com PC, o que justificou a realização da ACF associada à VED. Este estudo coincide com a lite$\operatorname{ratura}^{(4,6,7,26)}$, ao verificar que essas avaliações são complementares, sendo essenciais no estudo da disfagia orofaríngea neurogênica.

Houve limitações referentes à dificuldade na cooperação da população para realizar a VED, em decorrência do incômodo gerado pelo exame às crianças com PC.

\section{CONCLUSÃO}

Independentemente do tipo de PC, ou da idade cronológica da criança, haverá manifestações disfágicas na dinâmica da deglutição.

Quanto à administração das consistências alimentares e à presença de manifestações disfágicas, observou-se que o escape anterior e posterior do alimento; e a presença de sinais clínicos de penetração laríngea e/ou de aspiração traqueal, estiveram associados com a consistência líquida. Em contrapartida, as consistências pastosa grossa e sólida relacionaram-se com o aumento do trânsito oral e faríngeo, a diminuição da formação do bolo alimentar e a presença de resíduos após a deglutição na cavidade oral e estruturas faríngeas.

Conclui-se que são complementares e essenciais as avaliações clínica fonoaudiológica e videoendoscópica da deglutição no diagnóstico das disfagias orofaríngeas, além auxiliarem no prognóstico e processo terapêutico. 


\begin{abstract}
Purpose: To investigate the main manifestations of dysphagia in clinical and instrumental evaluation of swallowing in children with cerebral palsy. Methods: Cross-sectional study of 50 children with cerebral palsy. The data collection consisted of clinical speech-language pathology evaluation and fiberoptic endoscopic evaluation of swallowing for the detection of the main manifestations of dysphagia present in oral preparatory, oral and pharyngeal phases of swallowing, with different food consistencies and types of cerebral palsy. Results: There was no significant influence of age and type of cerebral palsy over the presence of dysphagia manifestations. The main manifestations in the clinical evaluation were: anterior premature spillage for liquids (70\%); cough during swallowing of liquids (60\%); decreased solid bolus formation (57.9\%); presence of food residues on the oral cavity after deglutition of solids (47.4\%); extended oral transit of thick food (47.1\%); and presence of wet voice after swallowing of liquids (30\%). The main manifestations of dysphagia in instrumental evaluation were: extended pharyngeal transit of thick food (79\%); posterior premature spillage for liquids $(77.5 \%)$; presence of thick food residues on the pharyngeal cavity after swallowing (58.8\%); laryngeal penetration for liquids (37.5\%), and tracheal aspiration for liquids (20\%). Laryngeal sensibility was altered in only $16 \%$ of the sample. Conclusion: Clinical and instrumental evaluations of swallowing are complementary and essential in the diagnosis of oropharyngeal dysphagia in children with cerebral palsy.
\end{abstract}

Keywords: Deglutition; Cerebral palsy/complications; Deglutition disorders/diagnosis; Pharynx/physiopathology; Child

\title{
REFERÊNCIAS
}

1. Matsuo K, Palmer JB. Anatomy and physiology of feeding and swallowing: normal and abnormal. Phys Med Rehabil Clin N Am. 2008;19(4):691-707, vii.

2. Miller AJ. The neurobiology of swallowing and dysphagia. Dev Disabil Res Rev. 20 08;14(2):77-86.

3. Gisel E. Interventions and outcomes for children with dysphagia. Dev Disabil Res Rev. 2008;14(2):165-73.

4. Santini CS. Disfagia neurogênica. In: Furkim AM, Santini CS. Disfagias orofaríngeas. 2a. ed. Carapicuíba: Pró-Fono; 2004. p. 19-34.

5. Aviv JE, Murry T, Zschommler A, Cohen M, Gartner C. Flexible endoscopic evaluation of swallowing with sensory testing: patient characteristics and analysis of safety in 1,340 consecutive examinations. Ann Otol Rhinol Laryngol. 2005;114(3):173-6.

6. Prasse JE, Kikano GE. An overview of pediatric dysphagia. Clin Pediatr (Phila). 2009;48(3):247-51.

7. Marrara JL, Duca AP, Dantas RO, Trawitzki LV, Lima RA, Pereira JC. Deglutição em crianças com alterações neurológicas: avaliação clínica e videofluoroscópica. Pró-Fono. 2008;20(4):231-6.

8. Bax M, Goldstein M, Rosenbaum P, Leviton A, Paneth N, Dan B, Jacobsson B, Damiano D; Executive Committee for the Definition of Cerebral Palsy. Proposed definition and classification of cerebral palsy, April 2005. Dev Med Child Neurol. 2005;47(8):571-6. Review.

9. Rosenbaum P, Paneth N, Leviton A, Goldstein M, Bax M, Damiano D, et al. A report: the definition and classification of cerebral palsy April 2006. Dev Med Child Neurol Suppl. 2007;109:8-14. Erratum in: Dev Med Child Neurol. 2007;49(6):480.

10. Calis EA, Veugelers R, Sheppard JJ, Tibboel D, Evenhuis HM, Penning C. Dysphagia in children with severe generalized cerebral palsy and intellectual disability. Dev Med Child Neurol. 2008;50(8):625-30.

11. Lach LM, Kohen DE, Garner RE, Brehaut JC, Miller AR, Klassen AF, Rosenbaum PL. The health and psychosocial functioning of caregivers of children with neurodevelopmental disorders. Disabil Rehabil. 2009;31(9):741-52.

12. Redstone F, West JF. The importance of postural control for feeding. Pediatr Nurs. 2004;30(2):97-100.

13. Val DC, Limongi SC, Flabiano FC, Silva KC. Sistema estomatognático e postura corporal na criança com alterações sensório-motoras. Pró-Fono. 2005;17(3):345-54.

14. Vivone GP, Tavares MM, Bartolomeu RS, Nemr K, Chiappetta AL. Análise da consistência alimentar e tempo de deglutição em crianças com paralisia cerebral tetraplégica espástica. Rev CEFAC. 2007;9(4):504-11.
15. Andreghetti SV, Furquim AM. O estudo videofluoroscópico da aspiração traqueal em pacientes portadores de paralisia cerebral do tipo quadriparesia espástica com queixa de deglutição. Rev CEFAC. 2003;5(2):143-8.

16. Helfrich-Miller KR, Rector KL, Straka JA. Dysphagia: its treatment in the profoundly retarded pacient with cerebral palsy. Arch Phys Med Rehabil. 1986;67(8):520-5.

17. Plant RL. Anatomy and physiology of swallowing in adults and geriatrics. Otolaryngol Clin North Am. 1998;31(3):477-88.

18. Buchholz DW, Bosma JF, Donner MW. Adaptation, compensation, and decompensation of the pharyngeal swallow. Gastrointest Radiol. 1985;10(3):235-9.

19. Pikus L, Levine MS, Yang YX, Rubesin SE, Katzka DA, Laufer I, Gefter WB. Videofluoroscopic studies of swallowing dysfunction and the relative risk of pneumonia. AJR Am J Roentgenol. 2003;180(6):1613-6.

20. Daniels SK, Corey DM, Hadskey LD, Legendre C, Priestly DH, Rosenbek JC, Foundas AL. Mechanism of sequential swallowing during straw drinking in healthy young and older adults. J Speech Lang Hear Res. 2004;47(1):33-45.

21. Groher ME, Crary MA, Carnaby Mann G, Vickers Z, Aguilar C. The impact of rheologically controlled materials on the identification of airway compromise on the clinical and videofluoroscopic swallowing examinations. Dysphagia. 2006;21(4):218-25.

22. Willging JP, Thompson DM. Pediatric FEESST: fiberoptic endoscopic evaluation of swallowing with sensory testing. Curr Gastroenterol Rep. 2005;7(3):240-3.

23. Jones B, Donner MW. Examination of the patient with dysphagia. Radiology. 1988;167(2):319-26. Erratum in: Radiology. 1991;179(3):881.

24. Jones B. Adaptation, compensation, and decompensation In: Jones B. Normal and abnormal swallowing: imaging in diagnosis and therapy. 2a ed. New York: Springer; 2003. p. 1-9.

25. Lucchi C, Flório CP, Silvério CC, Reis TM. Incidência de disfagia orofaríngea em pacientes com paralisia cerebral do tipo tetraparéticos espásticos institucionalizados. Rev Soc Bras Fonoaudiol. 2009;14(2):172-6

26. Furkim AM, Behlau MS, Weckx LL. Avaliação clínica e videofluoroscópica da deglutição em crianças com paralisia cerebral tetraparética espástica. Arq Neuropsiquiatr. 2003;61(3):611-6. 